International Journal of Zoological Investigations

Contents available at Journals Home Page: www.ijzi.net

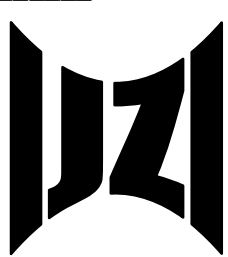

ISSN: 2454-3055

\title{
Potential of Fenoxycarb (a Juvenile Hormone Analogue) on the Growth Duration and Longevity of Adults of Rice Moth, Corcyra cephalonica Staint. (Lepidoptera:Pyralidae) Exposed as Second Instar Larvae
}

\section{Tiwari Shri Krishna}

Department of Zoology, D.D.U. Gorakhpur University, Gorakhpur- 273009, Uttar Pradesh, India

Received: $11^{\text {th }}$ May, 2020

Accepted: $16^{\text {th }}$ June, 2020

Published online: $23^{\text {rd }}$ June, 2020

https://doi.org/10.33745/ijzi.2020.v06i01.015

\begin{abstract}
Corcyra cephalonica larvae, at second instar level, were exposed to $0.001,0.005,0.01,0.05,0.10,0.50$ and $1.00 \mathrm{ppm}$ concentrations of fenoxycarb. It was observed that increased concentrations of fenoxycarb caused a significant $(\mathrm{P}<0.01)$ enhancement in growth duration, due to its juvenoid action, and an insignificant reduction in adult longevity in both the sexes of this pest.
\end{abstract}

Keywords: Corcyra cephalonica, Fenoxycarb, Growth duration, Longevity

Citation: Tiwari S.K.: Potential of fenoxycarb (a juvenile hormone analogue) on the growth duration and longevity of adults of rice moth, Corcyra cephalonica staint. (Lepidoptera:Pyralidae) exposed as second instar larvae. Intern. J. Zool. Invest. 6 (1): 187-195, 2020. https://doi.org/10.33745/ijzi.2020.v06i01.015

\section{Introduction}

Control of insect pests is a puzzling problem since many decades. Corcyra cephalonica Stainton, commonly known as rice moth, is a severe pest of stored cereals and cereal products in Asia, Africa, Europe, North America and other tropical and subtropical regions of the world. Its larval stages cause serious damage to rice, gram, sorghum, maize, groundnut, cotton seeds, peanuts, linseeds, raisins, nutmeg, chocolates and milled products (Ayyar, 1919, 1934; Chittenden, 1919; Piltz, 1977; Atwal, 1976).

Usually, the control measures in stores are based on fumigation with chemicals like hydrogen phosphate. Residues and insect resistance are reasons for potentially limiting the use of fumigation with chemicals in near future. In recent years, there has been great concern over the toxicity of pesticides on non-target organisms and the environment. 
Although, chemical pesticides are invaluable in controlling insect populations both in the field and storage, their indiscriminate use has resulted in the destruction of beneficial insects and has caused environmental hazards (Parween, 1997; Masner et al., 1980; Retnakaran et al., 1985). Moreover, insecticide resistance has already developed in many insects which is now a great concern in postharvest ecosystems throughout the word (Thind and Edwards, 1986; Arthur and Phillips, 2003) . Persistent use of synthetic organic insecticides affect immune system of insects, develop resistance and of course pollute our own environment due to nonbiodegradability, biomagnification and toxicity to non-target organisms.

In such condition, there is a need for new alternatives to traditional insecticides used in stored product pest management (Metwally and Landa, 1972; Atwal, 1976 b; Edwards and Short, 1984; Arthur and Phillips, 2003). In this regard, the insect growth regulators ( Gadenne et al., 1990) which mimic insect's hormone and regulate the insect population through the disruption of moulting and metamorphosis (Williams, 1967; Parthasarathy et al., 2012) have captured the interest of entomologists. The first use of IGRs (Insect Growth Regulators) against stored product pests was reported by Thomas and Bhatnagar-Thomas, 1968) but they have only been tested on a small number of insect species. The term IGR was designed by Stall (1975) to describe a class of bio-rational compounds. Through selectivity of action, these compounds appear to fit the requirements for "Third Generation Pesticides" (Williams, 1967) that disrupt the normal development of several species of insects (Henrick et al., 1973). These compounds are highly effective against various insects attacking stored products and other pests that have become resistant to organic insecticides. Meanwhile, all these compounds are less toxic to mammals and non-target organisms because of their nontoxic effect and their quick disintegrating abilities (Carter, 1975; Staal, 1975; Oberlander et al., 1978, 1979; Ishaaya et al., 1987; Ishaaya and Horowtz, 1998; Kostyukovsky et al., 2000; Parthasarathy et al., 2012).

Pener and Dhadialla (2012) proposed the use of term "Insect Growth Disrupters" instead of "Insect Growth Regulators". Williams (1967) made the famous statement, "Third Generation Pesticides" in describing the use of JHs as environmentally safe control agents to which the insect will be unable to develop resistance. The development of highly potent synthetic analogues of $\mathrm{JH}$, which were several fold more active than the native hormone, gave credence to William's claim. Role of JHAs on the growth duration of insects have also been reported in different insect species like C. cephalonica exposed to methoprene (Deb and Chakravotry, 1985); resistant strains of Tribolium castaneum treated with methoprene (Kostyukovsky et al., 2000); Ploida interpuctella following exposure of pyriproxyfen (Ghasemi et al., 2010); Eurygaster integriceps treated with pyriproxyfen (Mojaver and Bandani, 2010) and cowpea weevil, Callosobruchus maculatus exposed to hydroprene (Rup and Chopra, 1984). Adult longevity has also been found to be influenced by the exposure of IGRs as observed in case of Tenebrio molitor treated with diflubezuron (Soltani, 1984); C. cephalonica, Callosobruchus maculatus and Tribolium castaneum exposed to methoprene 
and hyroprene (Murali Baskaran and Janarthanan, 1991); Rhyzopertha dominica, Sitophilus oryzae and Tribolium castaneum following treatment with methoprene and pyriproxyfen (Kostyukovsky et al., 2000); Plodia interpuctella treated with pyriproxyfen (Ghasemi et al., 2010); Eurygaster integriceps exposed to pyriproxyfen (Mojaver and Bandani, 2010); Tribolium castaneum and Tribolium confusum following treatment with methoprene (Tucker et al., 2014).

Scientific contribution in relation to juvenile hormone analogues (JHAs) influencing developmental stages of $C$. cephalonica has been explored (Singh, 2014), but their potential on growth duration and adult longevity is completely wanting. The acquisition of such knowledge in this area becomes essential for a comprehensive appreciation of the physiological and ecological relationship that exists between this pest and its host material (stored cereals and cereal commodities). This knowledge in turn, is likely to generate new insights into divising ways and means for controlling $C$. cephalonica, by disrupting its metabolic frame work so that evolution of a new generation of this pest for the eventual establishment on stored cereals and cereal products can be considerably restricted. Hence, as an objective of such programme the present work for the first time, has been designed and conducted to examine the impact of a juvenile hormone analogue (JHA) i.e. fenoxycarb on the growth duration and adult longevity of rice moth, $C$. cephalonica.

\section{Materials and Methods}

Corcyra cephalonica Stainton adults were obtained from already existing laboratory stock culture maintained on normal dietary medium composed of coarsely ground jowar (Sorghum vulgare) mixed with $5 \%(\mathrm{w} / \mathrm{w})$ powdered yeast inside large glass containers (150 $\mathrm{mm}$ diameter, $200 \mathrm{~mm}$ height) at temperature $26 \pm 1 \mathrm{C}$, relative humidity (R.H.) $93 \pm 5 \%$ and a light regime of $12 \mathrm{~h}$ light and $12 \mathrm{~h}$ darkness. Such a standard culture was maintained throughout the year.

From the above culture whenever needed, newly emerged males and females were transferred to oviposition glass chambers (35 $\mathrm{mm}$ diameter, $200 \mathrm{~mm}$ height). Since $C$. cephalonica individuals do not feed during their adult stage, no food was provided to them during their confinement in these vessels. Eggs laid by the females were collected and then placed in glass chambers (consisting of $250 \mathrm{ml}$ beakers) with the help of zero number camel hair brush for hatching.

Fenoxycarb ethyl[2-(4-phenoxy-phenoxy)ethyl]carbamate, molecular formula$\mathrm{C}_{17} \mathrm{H}_{19} \mathrm{NO}_{4}$, a non terpenoid juvenile hormone analogue, P-686N, Lot-20071 used throughout the experiment, was obtained from AccuStandard, New Haven, CT 06513, USA.

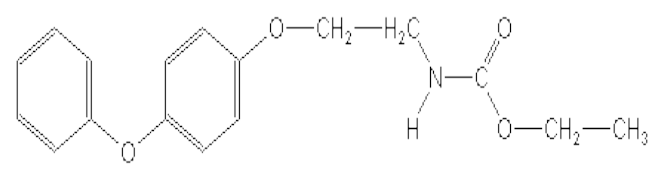

Fenoxycarb

Different concentrations of fenoxycarb, in dietary media, were prepared. For this purpose, a stock solution of known concentration of this JHA was prepared by dissolving it in acetone and then adjusted via serial dilutions to achieve its required concentrations. Then, required volume of different concentrations of fenoxycarb was thoroughly mixed with the required quantity of normal food (roughly ground jowar mixed 
with $5 \% \mathrm{w} / \mathrm{w}$ yeast powder) to get different desired concentrations i.e. 0.001, 0.005, 0.01, $0.05,0.10,0.50$ and $1.00 \mathrm{ppm}$ of fenoxycarb in dietary media. This treated food was then air dried at room temperature to eliminate completely the acetone. For control purposes, the normal food was thoroughly mixed with a required volume of acetone similar to that of treated food and then air dried in the same way.

For evaluation of growth duration, at each concentration of fenoxycarb, freshly hatched larvae of $C$. cephalonica were allowed to feed on anormal dietary medium for exactly nine days and on the $10^{\text {th }}$ day, 25 second instar larvae were transferred to each rearing chamber containing $50 \mathrm{~g}$ of dietary medium mixed and treated separately with different known concentrations of fenoxycarb. On completion of life-cycle, when adults emerged , growth duration of both sexes were recorded (Table 1). The longevity of adults emerged from these treated food were also recorded from day of emergence to day of death of males and females (Table 1).

Experiments were replicated six times and the values have been expressed as mean \pm SEM in days. Straight line regression equation was applied between different concentrations of fenoxycarb and their corresponding growth duration of males and females and longevity of adults to observe the significant correlation (Sokal and Rohlf, 1969).

\section{Results and Discussion}

In case of second instar larval treatment the growth duration of control male moth was found to be $37.83 \pm 0.70$ days and growth duration of female moth was $40.83 \pm 0.87$ days which significantly increased with increase in concentrations of fenoxycarb (Table 1). At $0.001 \mathrm{ppm}$ concentration the growth duration of male and female was 41.33 \pm 0.67 and $43.83 \pm 1.01$ days, respectively which increased to $85.17 \pm 1.81$ and $86.50 \pm$ 1.98 days for males and females, respectively at $0.10 \mathrm{ppm}$ concentration of fenoxycarb.

It is also notable that at 0.50 and $1.00 \mathrm{ppm}$ concentrations of fenoxycarb, larval period was much prolonged which lead to production of giant larvae, supernumerary larvae and their average life-span was recorded to be $90.17 \pm 2.41$ and $103.67 \pm 2.95$ days, respectively. These larvae did not emerged as adults so whether these were male or female could not be determined.

In case of control males, the adult longevity was found to be $13.67 \pm 0.33$ days, which significantly reduced to $11.50 \pm 0.43$ days at $0.001 \mathrm{ppm}$ and $5.50 \pm 1.48$ days at $0.10 \mathrm{ppm}$ concentrations of fenoxycarb, respectively. The longevity of control females, was recorded as $8.67 \pm 0.33$ days, which also reduced with increasing concentrations of fenoxycarb. At $0.001 \mathrm{ppm}$ concentration of fenoxycarb the longevity of female was $8.00 \pm$ 0.52 days which reduced to $4.17 \pm 1.05$ days at $0.10 \mathrm{ppm}$ concentration of fenoxycarb. In addition at 0.05 and $0.10 \mathrm{ppm}$ concentrations of fenoxycarb many of emerged adults were died within $24 \mathrm{~h}$ of their emergence.

JHA as a whole distrupt insect metamorphosis, hence, the developmental time for pupation and adult eclosion are considerably increased. 
Table 1: Effect of fenoxycarb on growth duration and longevity of adults of rice moth, C. cephalonica exposed as second instar larvae

\begin{tabular}{ccccc}
\hline $\begin{array}{c}\text { Fenoxycarb } \\
\text { concentration } \\
\text { (ppm) }\end{array}$ & $\begin{array}{c}\text { Growth duration\# } \\
\text { of males (Days) }\end{array}$ & $\begin{array}{c}\text { Growth duration\# } \\
\text { of females (Days) }\end{array}$ & $\begin{array}{c}\text { Adult longevity\# } \\
\text { of males (Days) }\end{array}$ & $\begin{array}{c}\text { Adult longevity\# } \\
\text { of females (Days) }\end{array}$ \\
\hline Control & $37.83 \pm 0.70$ & $40.83 \pm 0.87$ & $13.67 \pm 0.33$ & $8.67 \pm 0.33$ \\
0.001 & $41.17 \pm 0.87^{\mathrm{c}}$ & $42.83 \pm 0.60$ & $11.50 \pm 0.43^{\mathrm{b}}$ & $8.00 \pm 0.52$ \\
0.005 & $43.50 \pm 1.52^{\mathrm{b}}$ & $46.83 \pm 1.60^{\mathrm{b}}$ & $11.33 \pm 0.49^{\mathrm{b}}$ & $7.33 \pm 0.49^{\mathrm{c}}$ \\
0.01 & $51.83 \pm 1.58^{\mathrm{a}}$ & $53.83 \pm 1.14^{\mathrm{a}}$ & $10.33 \pm 0.49^{\mathrm{a}}$ & $6.67 \pm 0.56^{\mathrm{c}}$ \\
0.05 & $78.17 \pm 1.36^{\mathrm{a}}$ & $80.17 \pm 0.98^{\mathrm{a}}$ & $6.00 \pm 1.67^{\mathrm{b}}$ & $4.50 \pm 1.15^{\mathrm{b}}$ \\
0.10 & $85.17 \pm 1.81^{\mathrm{a}}$ & $86.50 \pm 1.98^{\mathrm{a}}$ & $5.50 \pm 1.48^{\mathrm{a}}$ & $4.17 \pm 1.05^{\mathrm{b}}$ \\
0.50 & $90.17 \pm 2.41^{\mathrm{a}^{*}}$ & $90.17 \pm 2.41^{\mathrm{a}^{*}}$ & - & - \\
1.00 & $103.67 \pm 2.95^{\mathrm{a}^{*}}$ & $103.67 \pm 2.95^{\mathrm{a}^{*}}$ & - & - \\
\hline
\end{tabular}

\# Values are expressed as mean \pm SEM of six replicates.

$\mathrm{a}, \mathrm{b}$ and $\mathrm{c}$ significantly different $\mathrm{p}<0.001, \mathrm{p}<0.01$ and $\mathrm{p}<0.05$, respectively compared with control when $\mathrm{t}$ test was applied.

Straight line regression equation was applied between different concentrations of fenoxycarb and their corresponding growth duration of males and females and longevity of adults to observe the significant correlation:

Growth duration of males

Growth duration of

$\mathrm{y}=42.98+480.53 \mathrm{x}$

$r=0.95$

$\mathrm{p}<0.01$

females

$\mathrm{y}=45.54+468.43 \mathrm{x} ;$

$r=0.95$

$\mathrm{p}<0.01$

Longevity of male adults

$\mathrm{y}=11.76-73.74 \mathrm{x} ;$

$\mathrm{r}=-0.90$

$r=-0.90$

$\mathrm{p}<0.05$

$\mathrm{y}=7.71-41.71 \mathrm{x}$;

$\mathrm{p}<0.05$

* Larval tenure since adults did not emerge, so these concentrations have not been accounted in correlation.

Due to prolongation of larval period there was delayed pupation or adult emergence. Hence, overall duration of growth period was found to increase with increase in concentration of fenoxycarb in this experiment. A short exposure to IGRs at definite instars of insect larvae would not affect the developmental time but a long exposure usually showed profound effects (Mian and Mulla, 1982). In the present investigation, application of fenoxycarb to the first instar larval stage of $C$. cephalonica caused significant influence on its growth duration (the time elapsing between egg laying to adult emergence) (Singh, 2014; Tiwari, 2018). Enhancement in the growth duration was observed when first instar larvae were treated with higher concentration of fenoxycarb (Singh, 2014)). Similarly, methoprene at a dose level of 10 and $100 \mu \mathrm{g}$ also influenced the time required for emergence of $C$. cephalonica when treated at 0-24 h old larval stage (Deb and Chakravorty, 1985) but methoprene at a concentration of $0.5 \mathrm{ppm}$ was found to be poorly effective in case of resistant strains of Tribolium castaneum while its $0.1 \mathrm{ppm}$ concentration 
was not effective (Kostyukovsky et al., 2000). Pyriproxyfen, a fenoxycarb derivative juvenile hormone analogue, with increased concentrations also increased growth duration effectively in case of the Plodia interpunctella even at poor concentrations (Ghasemi et al., 2010) but the same compound was found to be poorly effective in case of Eurygaster integriceps (Mojaver and Bandani, 2010). Prolongation in the developmental period of Callosobruchus maculatus has also been reported following exposure of hydroprene (Rup and Chopra, 1984). From these reports, it is evident that toxicity of IGRs to developmental stages of insects is species specific. Since, in the present investigation, the duration of fenoxycarb exposure to the second instar larvae was less in comparison to that of first instar treated larvae (Tiwari, 2018), hence, the growth duration was found to be maximum in case of first instar treated larvae. Thus, even at the same concentration of fenoxycarb the growth duration of the insect increases with the decrease in the age related duration of exposure. Fenoxycarb when exposed to second and third instar larvae of Ostrinia nubilalis had no significant effects on the duration of these instars while the duration of the first instar treated larvae increased significantly (Gadenne et al., 1990).

Prolongation of larval period by application of JHA in food medium was reported by Kostyukovsky et al. (2000) and Ghasemi et al. (2010) at different concentrations. Methoprene at dose levels of 10 and $100 \mu \mathrm{g}$ also influenced the time required for emergence of C. cephalonica when treated at 0-24 h old larval stage (Deb and Chakravorty, 1984). It is noteworthy that in the present investigation growth duration of female was slightly longer than male in control as well as in treated group. In all the instars maximum larval duration was achieved at $1.00 \mathrm{ppm}$ concentration of fenoxycarb which was $105.67 \pm 3.51$ days ( Tiwari, 2018) in case of first instar and 103.67 \pm 2.95 days in second instar treated larvae. The intensity of increase in larval duration was decreased with older instar larval treatments as at $1.00 \mathrm{ppm}$ concentration of fenoxycarb larval duration was $102 \pm 2.66$ and $97.17 \pm 2.96$ days for third and fourth instars, respectively (Singh, 2014). These larvae remained as larvae and after variable periods they stopped feeding and movement, turned black and eventually died.

In the present work, the longevity of $C$. cephalonica adults emerged from fenoxycarb treated larvae was significantly reduced. In the control, adult longevity was $13.50 \pm 0.42$ days for males and $8.50 \pm 0.43$ days for females whereas the longevity of adults emerged from firstinstar larvae treated with $0.10 \mathrm{ppm}$ fenoxycarb was $5.00 \pm 0.86$ days for males and $4.00 \pm 0.97$ days for females (Singh, 2014; Tiwari,2018). Similar findings were reported following the exposure of pyriproxyfen on Plodia interpunctella exposed to $0.3 \mathrm{ppm}$ of pyriproxyfen (Ghasemi et al., 2010) and on Eurygaster integriceps (Mojaver and Bandani, 2010). When 6-day-old Tenebrio molitor pupae were treated with $0.1 \mu \mathrm{g} \mathrm{JHA}$, adult life span was reduced to 4-6 days against the normal i.e. 14-16 days (Metwally and Landa, 1972). Methoprene and hydroprene also at the concentrations of 0.25 , 0.5, 1 and 2 ppm reduced longevity in case of Corcyra cephalonica, Callosobruchus maculatus and Tribolium castaneum (Murali Baskaran and Janarthanan, 1991). However, methoprene and pyriproxyfen act in a different way i.e. they have no effect on the 
life-span of Rhyzopertha dominica and Sitophilus oryzae, but have a profound effect on Tribolium castaneum (Kostyukovsky et al., 2000).

IGRs are not toxic to the adults themselves but long exposure to the early larval stages cause disruptions in the organ systems or interrupts adult ecdysis and reduces the adult life-span (Mondal and Parween, 2000). Fenoxycarb reduced adult life-span in other species than did malathion in stored rice (Cogburn, 1988). Tucker et al. (2014) also determined the ability of Tribolium castaneum and Tribolium confusum to develop successfully when treated at late instar larval stage with methoprene. They stated that for Tribolium castaneum, survival time for individuals exposed to methoprene was shorter than those exposed to control individuals, but the difference was singnificant for Tribolium confusum.

Adult longevity also depends on healthy immature stages. Digestive disorders such as starvation, disturbance in metabolism, degeneration of peritrophic membrane and accumulation of faecal materials at the hind gut may be the cause of untimely adult mortality as a result of BPU exposure (Soltani, 1984; Parween, 1997). Mean longevity of mated adults treated with $10 \mathrm{ppm}$ IGR (juvenoids and BPUs) was decreased compared to untreated and unmated adults in Plodia interpunctella whereas the tested IGRs lengthened life-span of the mated adults of $C$. cephalonica (Fajardo and Morallo-Rejesus, 1980). The toxic effect of fenoxycarb might be responsible for the reduction in the longevity of adults from treated culture. The longevity of adults has also been found to be increased with the increase in the age related duration of treatment. Adults emerged from first instar larvae treated with fenoxycarb survived for shortest time in comparison to those adults emerged from second, third and fourth instars treated larvae (Singh, 2014, Tiwari, 2018).

\section{Conclusion}

It is concluded that initial stage of $C$. cephalonica larva must be treated with fenoxycarb to facilitate lenthy duration of exposure for the effective control of this pest in particular and lepidopterous pests in general.

\section{Acknowledgement}

Author is highly thankful to AccuStandard, New Haven,CT 06513,USA for providing fenoxycarb, P-686N, Lot 20071.

\section{References}

Atwal AS. (1976) Agricultural Pests of India and SouthEast Asia. Kalyani Publishers, Delhi, pp: 502.

Ayyar TVR. (1919) Some insects recently noticed as injurious in South India. In: Report of the Proceedings of the Third Entomological Meeting. Fletcher, T.B. (ed.), Vol. 1, Pusa, Superintendent Government Printing, Calcutta, pp. 314-328.

Ayyar PNK. (1934) A very destructive pest of stored in South India, Corcyra cephalonica, Staint. (Lep.). Bull. Entomol. Res. 25(2): 155-169.

Carter DJ. (1975) Laboratory evaluation of three novel insecticides inhibiting cuticle formation against some susceptible and resistant stored products beetles. J. Stored Prod. Res. 11(3-4): 187-193.

Chittenden FH. (1919) The rice moth. U. S. Department of Agricultural, Bulletin No. 783. Washington (Govt. Print. Off.), pp. 15.

Cogburn RE. (1988 ) Fenoxycarb as a long term protectant for stored rough rice. J. Econ. Entomol. 81(2): 722-726.

Deb DC and Chakravorty S. (1985) Influence of additional corpora allata, juvenoids and antiallayotropin on the development and phenotypic changes of the rice moth Corcyra cephalonica (Stainton). Insect Sci. Appl. 6(1): 105-110.

Gadenne SC, Grenier S, Plantevin G and Mauchamp B. (1990) Effects of a juvenile hormone mimetic, fenoxycarb, on post-embryonic development of the 
European corn borer, Ostrinia nubilalis $\mathrm{Hbn}$. Experientia 46(7): 744-747.

Ghasemi A, Sendi J and Ghadamyari M. (2010) Physiological and biochemical effect of pyriproxyfen on Indian meal moth Plodia interpunctella (Hubner) (Lepidoptera: Pyralidae). J. Plant Prot. Res. 50 (4): 416-422.

Grenier S and Grenier AM. (1993) Fenoxycarb, a fairly new insect growth regulator: review of its effects on insects. Ann. Appl. Biol. 122(2): 369-403.

Henrick CA, Staal GB and Siddall JB. (1973) Alkyl 3, 7, 11-trimethyl-2, 4-dodecadienoates, a new class of potent insect growth regulators with juvenile hormone activity. J. Agric. Food Chem. 21: 354-359.

Ishaay I, Yablonski S and Ascher KRS. (1987) Toxicological and biochemical aspects of novel acylureas on resistant and susceptible strains of Tribolium castaneum. Proc. $4^{\text {th }}$ Int. Work Conf. Stored-Product Prot., Donahaye E. and S. Navarro, (eds.), Tel Aviv, Israel, pp. 613-622.

Ishaaya I and Horwitz AR. (1998) Insecticides with novel modes of action: an overview. In: Insecticides with Novel Modes of Action: Mechanisms and Application, Ishaaya, I. and Degheele D. (eds.), Springer, Berlin Heidelberg, pp. 1-24.

Kostyukovsky M, Chen B, Atsmi S and Shaaya E. (2000) Biological activity of two juvenoids and two ecdysteroids against three stored product insects. Insect Biochem. Mol. Biol. 30 (8-9): 891-897.

Masner P, Dorn S, Vogel W, Kalin M, Graf O and Gunthart E. (1980) Types of response of insects to a new IGR and to proven standard insecticides. In: Regulation of Insect Development and Behavior. Kloza, M. (ed.). Technical University Wroclaw Press, Poland. pp: 809-818.

Metwally MM and Landa V. (1972) Sterilization of the khapra beetle, Trogoderma granarium Everts, with juvenile hormone analogues. Z. Angew. Entomol. 72(1-4): 97-109.

Mian LS and Mulla MS. (1982) Residual activity of insect growth regulators against stored-product beetles in grain commodities. J. Econ. Entomol. 75: 599-603.

Mojaver M and Bandani AR. (2010) Effects of the insect growth regulator pyriproxyfen on immature stages of Sunn Pest, Eurygaster integriceps Puton (Heteroptera: Scutelleridae). Mun. Entomol. Zool. 5 (1): 187-197.

Moreno J, Hawlitzky N and Jimenez R. (1992) Effect of the juvenile hormone analog fenoxycarb on the last larval instar of Ephestia kuehniella Zell. (Lep., Pyralidae). J. Appl. Entomol. 114(1-5): 118-123.
Murali Baskaran KK and Janarthanan R. (1991) Effect of insect growth regulators on longevity of three species of stored products pests. Bull. Grain Technol. 29(1): 35-37.

Oberlander H, Nickle D, Silhacek DL and .Hagstrum DW. (1978) Advances in insect growth regulators research with grain insects. Symposium on the Prevention and Control of Insects in Stored Foods Products. Manhattan, Kansas. p. 247-263.

Parthasarathy R, Farkas R and Palli SR. (2012) Recent progress in juvenile hormone analogs (JHA) research. In: Advances in Insect Physiology, Insect Growth Disruptors. Dhadialla, T.S. (ed.), Vol. 43, pp. 353-436.

Parween S. (1997) Effect of triflumuron on the adult midgut of Tribolium castaneum Herbst (Coleoptera: Tenebrionidae). Univ. J. Zool. Rajshahi Univ. 16: 11-18.

Pener MP and Dhadialla TS. (2012) An overview of insect growth disruptors; applied aspects. Adv. Insect Physiol. 43: 1-162.

Piltz H. (1977) Corcyra cephalonica (Staint.) In: Disease Pests and Weeds tropical crops. Kranz, J., Schmutterer H. and Koch W. (eds.), Verlag Paul Parey, Berlin and Hamburg, pp. 439-440.

Retnakaran A, Granett J and Ennis TJ. (1985) Insect growth regulators. In: Comprehensive Insect Physiology, Biochemistry and Pharmacology. Kerkut, J.A. and Gilbert L.I. (eds.), Vol. 12, Pergamon Press, Oxford, pp. 529-601.

Rup P and Chopra PK. (1984) Effect of hydroprene on Callosobruchus maculates (F.) (Coleoptera: Bruchidae). J. Stored Prod. Res. 20(4): 229-232.

Singh A. (2014) Physiological and biochemical effects of fenoxycarb, a juvenile hormone analogue on the rice moth, Corcyra cephalonica Stainton (Lepidoptera: Pyralidae). Ph.D. Thesis, Dept. Zool. DDU Gorakhpur Uni., Gorakhpur 273009, U.P., India.

Sokal RR and Rohlf FJ. (1969) Introduction to Biostatistics. W.H.Freemann and Co.San Franscisco.

Soltani N. (1984) Effects of ingested diflubenzuron on the longevity and the peritrophic membrane of adult mealworms (Tenebrio molitor L.). Pest. Sci. 15(3): 221-225.

Staal GB. (1975) Insect growth regulators with juvenile hormone activity. Ann. Rev. Entomol. 20: 417-460.

Subramanyam BH and Hagstrum DW. (1995) Resistance measurement and management. In: Integrated Management of Insects in Stored Products, Subramanyam, Bh. and Hagstrum D.H. (eds.). Marcel Deckar, Inc. New York, pp: 331-397. 
Thind BB and Edwards JP. (1986) Laboratory evaluation of the juvenile hormone analogue fenoxycarb against some insecticide-susceptible and resistant stored products beetles. J. Stored Prod. Res. 22(4): 235-241.

Thomas PJ and Bhatnagar-Thomas PL. (1968) Use of a juvenile hormone analogue as insecticide for pests of stored grain. Nature 219: 949.

Tiwari SK. (2018) Effect of fenoxycarb on the growth duration and longevity of rice moth Corcyra cephalonica Staint. (Lepidoptera:Pyralidae) exposed as first instar larvae. J. Adv. Zool. 39 (1): 20-31.
Tucker AM, Campbell JF, Arthur FH and Zhu KY (2014) Horizontal transfer of methoprene by Tribolium castaneum (Herbst) and Tribolium confusum Jacquelin du Val. J. Stored Prod. Res. 57: 73-89.

Williams CM. (1956) The juvenile hormone of insects. Nature 178: 212-213.

Williams CM. (1967) Third generation pesticides. Sci. Am. 217: 13-17. 